\title{
A Novel Objective Quality Assessment for Super-Resolution Images
}

\author{
Lei Shu ${ }^{1}$, Yuming Fang ${ }^{1}$, Zhijun Fang ${ }^{1,2}$, Yong Yang ${ }^{1}$, Fengchang Fei $^{1}$ and \\ Naixue Xiong ${ }^{1, *}$ \\ ${ }^{1}$ School of Information Technology, Jiangxi University of Finance and Economics, \\ Nanchang, Jiangxi, China \\ ${ }^{2}$ College of Electronic and Electrical Engineering, Shanghai University of \\ Engineering Science, Shanghai, China \\ xiongnaixue@gmail.com
}

\begin{abstract}
A novel objective quality assessment method is proposed for super-resolution images in this manuscript. We not only estimate the preserved information of each spatial location in the super-resolution image by structural similarity, but also compute the local phase coherence $(L P C)$ with which we can detect the image blur in the super-resolution image. After the preserved structural information and blur information is obtained, an overall evaluation of visual quality of the super-resolution image can be computed. Experimental results show that the proposed objective quality assessment method can be used in the real applications with the original high-resolution images unavailable.
\end{abstract}

Keywords: image quality assessment; super-resolution imgae; structural similarity; local phase coherence; blur detection

\section{Introduction}

Image super-resolution, referring to the technique of generating a high-resolution (HR) image from low-resolution (LR) image(s), is keeping a hot topic since the early SR algorithm [1] was proposed. The image super-resolution method which is widely used in many applications such as medical imaging and video surveillance, is trying to provide the good visualization and additional information details by high resolution images. For example, a high resolution image can provide a better classification of regions in a remote sensing image or identify the edges of lesion area accurately in a medical healthcare system [2]. To get high-resolution images, one intuitive way is to reduce the pixel size on the sensor to increase the resolution of digital camera. However, this method needs replacing the sensor and thus expensive. Image super-resolution techniques by means of computer software, have been proved useful in many fields. Especially in the network era, the image super-resolution technology allows the usage of low resolution images for the fast transmission [3-4], while people can receive high resolution images in the client side [5]. Many super-resolution imaging frameworks have been proposed, including Iterated Back-Projection (IBP) [6], Maximum A Posteriori (MAP) [7], sparse representation [8], etc. Nevertheless, how to objectively evaluate the quality of super-resolution images remains a problem unsolved in the research community.

*Corresponding Author 
Image Quality Assessment (IQA) methods can be classified as subjective assessment and objective assessment, which denote quality evaluation by observers and by objective metrics, respectively. Since all the images have to be perceived by the Human Visual System (HVS) finally, subjective evaluation of visual image quality is the only "correct" metric. In practice, however, subjective evaluation is not only inconvenient and expensive, but also cannot be embedded into automatic systems to adjust themselves in real-time based on the feedback of output quality. Therefore, it is much desired to design objective image quality assessment metrics aiming to predict the visual quality of images consistent with that perceived by the humans.

According to the availability of an original image, traditional objective image quality assessment methods can be classified into three types [9]: full reference (FR) metrics which require the original image and distorted image, reduced-reference metrics (RR) which require part of the original image and the distorted image, and no reference metrics (NR) which require only the distorted image.

The mean squared error (MSE), as well as peak signal-to-noise ratio (PSNR), are the widely used FR metrics because they are simple and easy to implement and have clear physical meanings. However, they are failed to be consistent with perceived visual quality in many cases (e.g., [10-13]). The well-known Structure SIMilarity (SSIM) index [14] assumes that human visual system (HVS) tends to perceive the local structures in an image when evaluating its quality. Most FR metrics extract and compare the features of the original and distorted images to predict the visual quality of the distorted image. However, these IQA methods are not feasible to meet super-resolution image quality assessment task, since the image sizes are changed during image super-resolution. Furthermore, the original high resolution images are missing whenever the image superresolution methods are needed in real applications, which makes the FR metrics not working. So the objective image quality assessment metrics for super-resolution images are badly needed.

In this manuscript, we propose a novel objective quality assessment method for superresolution images based on structural similarity fidelity and image blur detection. Firstly, structural similarity metric (SSIM) [14] and scale invariant feature transform (SIFT) [15] are used to compute structural similarity fidelity of the super-resolution image. Then, an objective image sharpness metric is applied to compute the probability of image blur at each edge in the image by the Local Phase Coherence (LPC) [16]. At last, the results of structural similarity fidelity and image blur detection are combined to get the final quality score of super-resolution image.

The remainder of the paper is organized as follows. In Section II, the framework of the proposed method are introduced in detail. Section III presents the experimental results and Section IV provides the conclusion.

\section{The Proposed Method}

The general framework of the proposed method is depicted in Figure. 1. For each image pixel in the reference image (LR), we first find its best matching pixel in the superresolution image (HR). The SSIM measure is then calculated between the local regions surrounding and in the reference and super-resolution images, respectively. Then the SSIM map could be generated by applying this process to all pixels in the reference image. On the other hand, image blur will be detected at each edge in the super-resolution image by means of LPC. At last, the final quality score is obtained by combining the SSIM map and the image blur. Details about the proposed method are given in the following subsections. 


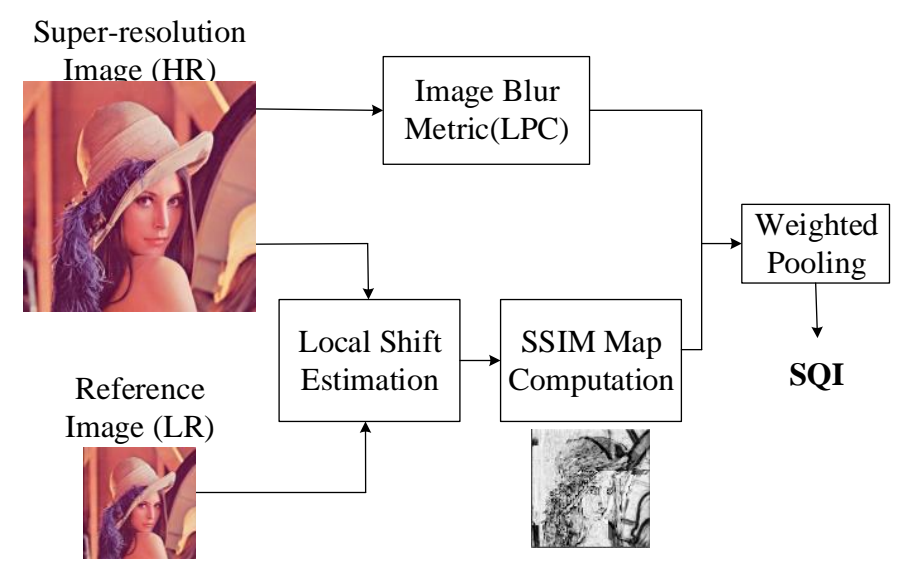

Figure 1. The Framework of the Proposed Method

\subsection{SSIM Map Estimation}

Because the reference and super-resolution images have different sizes and pixel correspondences are missing, the original SSIM algorithm [14] cannot be utilized directly. To address this problem, we need to apply shift estimation methods to build dense pixel correspondence between these images. The scale-invariant feature transform (SIFT) descriptors [15] achieves a promising matching across different image sizes or contents. SIFT flow has been proven to be effective in finding dense correspondence between two images [17]. As what we have done in our previous work [18], SIFT flow is employed to find the matching pixel in the super-resolution image for each pixel in the reference image.

Since the pixel correspondence between the reference image and the super-resolution image can be established through SIFT flow, the local structure in the corresponding regions can be compared. SSIM is used to predict the perceptual structural distortions of the distorted image [14-19]. In this paper, given two image patches $l$ and $h$ in the reference and super-resolution images, respectively, SSIM can be computed as follows.

$\operatorname{SSIM}(l, h)=\frac{\left(2 \mu_{l} \mu_{h}+C_{1}\right)\left(2 \sigma_{l h}+C_{2}\right)}{\left(\mu_{l}^{2}+\mu_{h}^{2}+C_{1}\right)\left(\sigma_{l}^{2}+\sigma_{h}^{2}+C_{2}\right)}$

where $\mu_{l}, \mu_{h}$ denote the local mean of $l$ and $h$, respectively; $\sigma_{l}^{2}, \sigma_{h}^{2}$ and $\sigma_{l h}$ denote the local variance of $l$ and $h$, and the local covariance between $l$ and $h$, respectively; $C_{1}$ and $C_{2}$ are small positive stability constants that account for the saturation effects of the visual system at low luminance and contrast [10]. Finally, the global SSIM map can be obtained by applying the local SSIM computation using a sliding window approach at every pixel across the LR image space. The mean SSIM (MSSIM) index is used to evaluate the overall structure similarity [10]

$\operatorname{MSSIM}(L, H)=\frac{1}{M} \sum_{j=1}^{M} \operatorname{SSIM}(l, h)$

where $L$ and $H$ are the reference (LR) and the super-resolution images, respectively; $l$ and $h$ are the image patches of $L$ and $H$; and $M$ is the number of patches of the image.

\subsection{Local Phase Coherence}

In the Section 2.1, we get the SSIM map for the structure similarity between the reference and the super-resolution images. However, it is not enough for super-resolution image quality assessment because something important is still missing. Super-resolution imaging algorithms are considered to recover the missing information in the image distortion progress. The super-resolution operation would cause different effects in the 
edge regions but little difference in the smooth regions. For example, many classical image interpolation methods generate blur in the edges, such as saw-tooth and ringing effect.

LPC [16] is a no-reference image blur metric which describes the alignment of local phase patterns in the scale-space in the vicinity of distinctive sharp image features [20]. In [21], a novel framework is proposed to compute LPC using samples arbitrarily extracted from the scale-space. Compare to the previous methods [22], the proposed framework is more flexible, and more importantly, can be made more space- and scale-localized, and thus reduces interference from nearby image features [21].

If we have a signal $f(x)$ created from a feature signal $f_{0}(x)$ but located near position $x_{0}$, i.e.. $f(x)=f_{0}\left(x-x_{0}\right)$, a general family of complex wavelet transform may be written as

$F(s, p)=\int_{-\infty}^{\infty} f(x) w_{s, p}^{*}(x) d x=\left[f(x) * \frac{1}{\sqrt{s}} g\left(\frac{x}{s}\right) e^{J w_{c} x / s}\right]_{x=p}$

where $s \in R^{+}$and $p \in R$ are the scale and the translation factor, respectively. The family of wavelets $w_{s, p}(x)$ are derived from a scaled and shifted mother wavelet $w(x)=g(x) e^{j w_{c} x}$ by

$w_{s, p}(x)=\frac{1}{\sqrt{s}} w\left(\frac{x-p}{s}\right)=\frac{1}{\sqrt{s}} g\left(\frac{x-p}{s}\right) e^{\frac{J w_{c}(x-p)}{s}}$

where $w_{c}$ is the center frequency of the modulated band pass filter, and $g(x)$ is a slowly varying, non-negative and symmetric envelop function.

Using the convolution theorem, and the shifting and scaling properties of the Fourier transform, we can derive:

$F(s, p)=\frac{1}{2 \pi \sqrt{s}} \int_{-\infty}^{\infty} F_{0}\left(\frac{w}{s}\right) G\left(w-w_{c}\right) e^{j w\left(p-x_{0}\right) / s} d w$

where $F(w), F_{0}(w)$ and $G(w)$ are the Fourier transforms of $f(x), f_{0}(x)$ and $\mathrm{g}(x)$, respectively. The phase of $F(s, p)$ depends on the nature of $F_{0}(w)$. If $F_{0}(w)$ is scale invariant, meaning that

$F_{0}(w / s)=K(s) F_{0}(w)$

where $K(s)$ is a real function of only s, but independent of $\omega$, then

$F(s, p)=\frac{K(s)}{2 \pi \sqrt{s}} \int_{-\infty}^{\infty} F_{0}(w) G\left(w-w_{c}\right) e^{j w\left(p-x_{0}\right) / s} d w$

Combining the case of an impulse and an ideal step edge, the general LPC relationship can be obtained

$\Phi(F(s, p)) \approx \widehat{\Phi}(s, p) \equiv \frac{w_{c}\left(p-x_{0}\right)}{s}+\frac{k \pi}{2}$

where $\mathrm{k}$ is an integer depending on the nature of the sharp feature.

If there are a set of samples $\Phi$, together with a corresponding set of weights $\mathrm{w}=\left[\mathrm{w}_{1}, \mathrm{w}_{2}, \ldots, \mathrm{w}_{\mathrm{N}}\right]^{\mathrm{T}}$, such that

$w^{T} \widehat{\Phi}=0$

then a simple measure of LPC strength can be defined by

$S_{L P C}=\cos \left(w^{T} \Phi\right)$ 
Since the LPC relationship holds at sharp image features only, it was conjectured that the visual perception of blur may be interpreted as a loss of LPC and the conjecture is partially supported by local phase statistics of sharp and blurred natural images [16-21].

The value of this LPC measure is bounded between -1 and 1 , and the maximal value is achieved when $\mathrm{w}^{\mathrm{T}} \Phi=0$, which is consistent with the phase relationship defined in (9). Substitute (8) into (9), then

$w_{c}\left(p-x_{0}\right)\left(\sum_{i=1}^{N} \frac{w_{i}}{s_{i}}\right)+\frac{k \pi}{2}\left(\sum_{i=1}^{N} w_{i}\right)=0$

In order for this to be true for all possible values of $w_{c}, p, x_{0}$ and $\mathrm{k}$, we would need the following simultaneous equations to be true

$\left\{\sum_{i=1}^{N} w_{i}=0\right.$

$\left\{\sum_{i=1}^{N}\left(w_{i} / s_{i}\right)=0\right.$

Without loss of generality, we assume $w_{1}=1$. This results in $N-1$ unknowns $\left(w_{2}, w_{3}, \ldots, w_{N}\right)$ with two equations. For convenience we take $N=3$, the solutions are unique and can be given by

$$
\left\{\begin{array}{l}
\mathrm{w}_{1}=1 \\
\mathrm{w}_{2}=\frac{\mathrm{s}_{2}\left(\mathrm{~s}_{3}-\mathrm{s}_{1}\right)}{\mathrm{s}_{1}\left(\mathrm{~s}_{2}-\mathrm{s}_{3}\right)} \\
\mathrm{w}_{3}=\frac{\mathrm{s}_{3}\left(\mathrm{~s}_{2}-\mathrm{s}_{1}\right)}{\mathrm{s}_{1}\left(\mathrm{~s}_{3}-\mathrm{s}_{2}\right)}
\end{array}\right.
$$

We choose $s 1$ to be the finest scale $\left(s_{1}=1\right)$ for maximal localization, and choose the other $s_{i}$ values to be evenly spaced in either linear or logarithm scale. Table I. shows the specific values of $\mathrm{d}$ and $\mathrm{r}$.

$\left[\begin{array}{l}s_{1} \\ s_{2} \\ s_{3}\end{array}\right]=\left[\begin{array}{c}1 \\ 1+d \\ 1+2 d\end{array}\right]$ or $\left[\begin{array}{l}s_{1} \\ s_{2} \\ s_{3}\end{array}\right]=\left[\begin{array}{c}1 \\ r \\ r^{2}\end{array}\right]$

Tabel 1. Weight Solutions for Three Scales

\begin{tabular}{|c|c|c|c|c|c|c|}
\hline & $s_{1}$ & $s_{2}$ & $s_{3}$ & $w_{1}$ & $w_{2}$ & $w_{3}$ \\
\hline & 1 & $1+\mathrm{d}$ & $1+2 \mathrm{~d}$ & 1 & $\begin{array}{c}- \\
2(1+\mathrm{d})\end{array}$ & $1+2 \mathrm{~d}$ \\
\hline $\mathrm{d}=1 / 4$ & 1 & $5 / 4$ & $3 / 2$ & 1 & $-5 / 2$ & $3 / 2$ \\
\hline $\mathrm{d}=1 / 2$ & 1 & $3 / 2$ & 2 & 1 & -3 & 2 \\
\hline $\mathrm{d}=1$ & 1 & 2 & 3 & 1 & -4 & 3 \\
\hline $\mathrm{d}=3 / 2$ & 1 & $5 / 2$ & 4 & 1 & -5 & 4 \\
\hline $\mathrm{d}=2$ & 1 & 3 & 5 & 1 & -6 & 5 \\
\hline & 1 & $r$ & $\mathrm{r}^{2}$ & 1 & $-(1+\mathrm{r})$ & $\mathrm{r}$ \\
\hline $\mathrm{r}=5 / 4$ & 1 & $5 / 4$ & $25 / 16$ & 1 & $-9 / 4$ & $5 / 4$ \\
\hline $\mathrm{r}=\sqrt{2}$ & 1 & $\sqrt{2}$ & 2 & 1 & $-1-\sqrt{2}$ & $\sqrt{2}$ \\
\hline $\mathrm{r}=\sqrt{3}$ & 1 & $\sqrt{3}$ & 3 & 1 & $-1-\sqrt{3}$ & $\sqrt{3}$ \\
\hline $\mathrm{r}=2$ & 1 & 2 & 4 & 1 & -3 & 2 \\
\hline $\mathrm{r}=\sqrt{5}$ & 1 & $\sqrt{5}$ & 5 & 1 & $-1-\sqrt{5}$ & $\sqrt{5}$ \\
\hline
\end{tabular}

If there is an input image whose sharpness needs to be assessed, it should be passed through a series of N-scale M-orientation log-Gabor filters without any subsequent downsampling process. This results in MN "subbands" and there are MN complex coefficients at each spatial location across all orientations and all scales. Let $\mathrm{c}_{\mathrm{ijk}}$ be the coefficient at 
the $\mathrm{i}$-th scale, the $\mathrm{j}$-th orientation and the k-th spatial location. Then the LPC strength at the $\mathrm{j}$-th orientation and the k-th location can be computed by

$S_{L P C}^{\{j, k\}}=\cos \left(w^{T} \Phi_{j}\right)=\cos \left(\sum_{i=1}^{N} w_{i} \Phi\left\{c_{i j k}\right\}\right)=\cos \left(\Phi\left\{\prod_{i=1}^{N} c_{i j k}^{w_{i}}\right\}\right)=\frac{\Re\left\{\prod_{i=1}^{N} c_{i j k}^{w_{i}}\right\}}{\left|\left\{\prod_{i=1}^{N} c_{i j k}^{w_{i}}\right\}\right|}$

where $\Re\{\cdot\}$ denotes the real part of a complex number. This LPC strength measure is combined at each spatial location $\mathrm{k}$ by a weighted average across all orientations, where the weights are determined by the magnitude of the first (finest) scale coefficient $c 1 j k$, so that the orientations that contain more energy are given higher importance:

$S_{L P C}^{\{k\}}$

$=\frac{\sum_{j=1}^{M}\left|c_{1 \mathrm{k}}\right| S_{L P C}^{\{j, k\}}}{\sum_{j=1}^{M}\left|c_{1 \mathrm{k} k}\right|+C}$

where a constant $\mathrm{C}$ is added to avoid instability when the magnitudes of the coefficients are near to 0 .

Let $\mathrm{S}_{\mathrm{LPC}}^{\{\mathrm{k}\}}$ for $\mathrm{k}=1,2, \ldots, \mathrm{K}$ be a collection of LPC values extracted from the LPC map, and let $S_{\mathrm{LPC}}^{\{(\mathrm{k})\}}$ for $\mathrm{k}=1,2, \ldots, \mathrm{K}$ denote the sorted LPC strength values such that $\mathrm{S}_{\mathrm{LPC}}^{\{(1)\}} \geq$ $S_{\mathrm{LPC}}^{\{(2)\}} \geq \ldots \geq \mathrm{S}_{\mathrm{LPC}}^{\{(\mathrm{K})\}}$. Then the overall LPC-based sharpness index (LPC-SI) is defined as [21]

$S_{\mathrm{LPC}}$

$=\frac{\sum_{k=1}^{K} u_{k} S_{\mathrm{LPC}}^{\{(k)\}}}{\sum_{k=1}^{K} u_{k}}$

where $\mathrm{u}_{\mathrm{k}}$ is the weight assigned to the $\mathrm{k}$-th ranked spatial LPC value and is computed as an exponentially decaying function given by

$u_{k}=\exp \left[-\left(\frac{k-1}{K-1}\right)\right.$

which gives a weight 1 to the highest LPC value and the decaying speed of the weights is controlled by the parameter $\beta_{\mathrm{k}}$. The parameters which used in computing LPC are set as $\mathrm{M}=8, \mathrm{~N}=3, \mathrm{~s}_{1}=1, \mathrm{~s}_{2}=3 / 2, \mathrm{~s}_{3}=2, \mathrm{C}=2$, and $\beta_{\mathrm{k}}=1 \mathrm{e}-4$.

\subsection{Quality Assessment Model}

The structural similarity measure MSSIM introduced in Section II-A and the image blur measure LPC described in Section II-B characterize different aspects of the visual quality of super-resolution images. Here, we combine them to obtain a joint measure, resulting in a Super-resolution image Quality Index (SQI)

$Q=a S^{\alpha}+$

$(1-a) P^{\beta}$

where $\mathrm{S}$ and $\mathrm{P}$ denote the MSSIM measure and LPC meausre, respectively; $0 \leq \mathrm{a} \leq 1$ adjusts the relative importance of these two components, and $\alpha$ and $\beta$ determine their sensitivities, respectively. The parameters in (19) are set empirically as a $=0.2, \alpha=0.3$, and $\beta=0.7$ in the experiments. 


\section{Experimental Results}

In this section, we conducted two experiments to evaluate the performance of the proposed SQI. The purpose of the first experiment is to compare the performance of different image super-resolution methods on test images from [23] with the original HR image available by PSNR, SSIM and SQI. The second experiment aims to test SQI on super-resolution images of natural scene without the original HR image.

\subsection{Experiment I}

In this experiment, we select 4 classical image super-resolution algorithms with 24 test images. To be fair for all test super-resolution algorithms, the scale factor is set to 2 . As Figure. 2 shows, the tests images are color images with the size of $512 * 512$. In order to simulate the natural imaging process, we carried out the shifting, blurring and downsampling process to the original images to obtain LR images, as Figure. 3 shows. Then different super-resolution algorithms are employed to LR images to get HR images. We select several classical image interpolation methods, such as Linear [24], Cubic [25], ICBI [26], and the sparse coding super-resolution method ( $\mathrm{ScSR})$ [8] which its matlab code is available on the website [27], to compare their performance on PSNR, SSIM and SQI, as Figure 4 shows.

We calculate the average score of PSNR, SSIM and SQI of all reconstructed HR images by the picked super-resolution algorithms, as Figure. 4 shows. We make no modification to the learning dictionary of original ScSR when we use ScSR to get HR images. Not surprisingly, we can easily find that the performance of Linear is worst in all metrics since Linear is the most naive one in these 3 interpolation algorithms, and it certainly not be better than the learning based algorithm. So, we only compare the other 3 algorithms in the rest of this experiment.

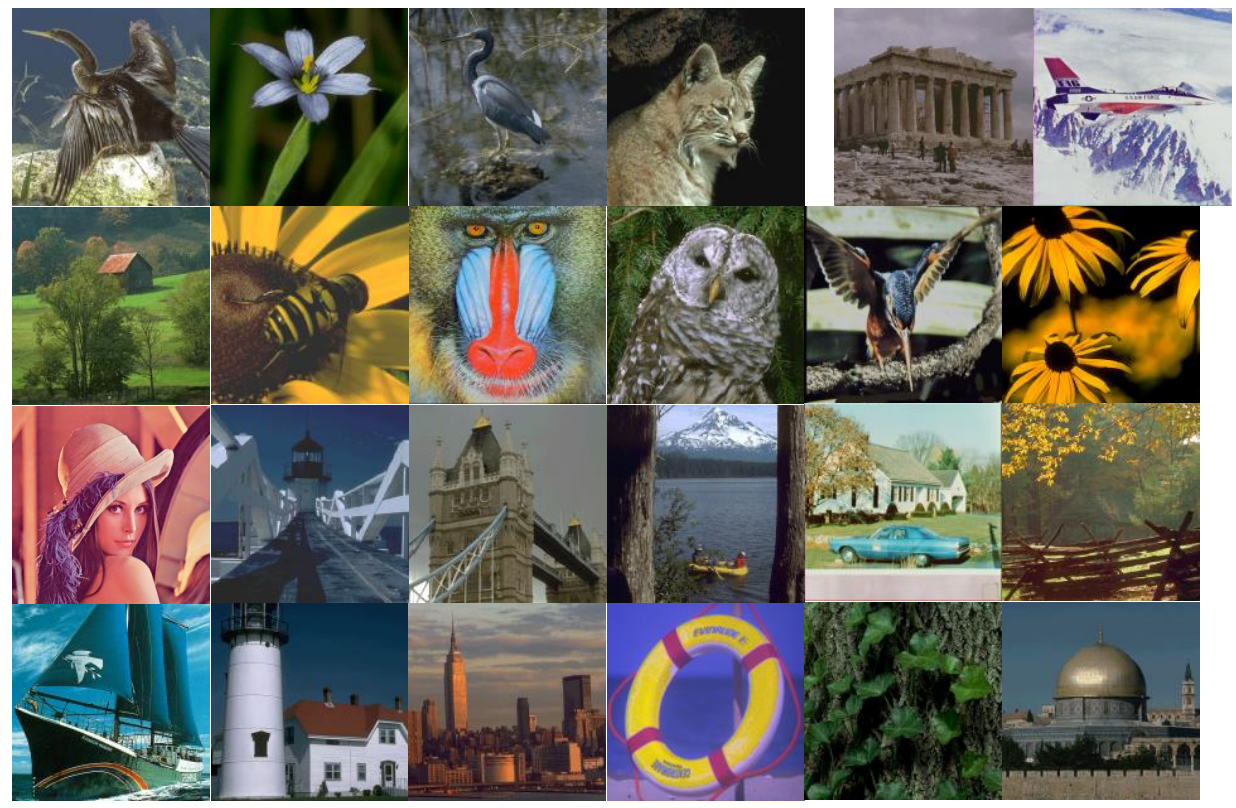

Figure 2. The Test Images of Experiment I

The ICBI achieves top place in PSNR, but the PSNR of ScSR and Cubic is very close behind. As we all know, the PSNR is not consist with the HSV in many cases. The ScSR achieves top place in SSIM and SQI. The difference of SSIM score is still very small which means it is a hard task to find the best one of these 3 methods by SSIM. The ScSR has obvious advantages in SQI average score. From this result, we can easily draw the conclusion that $\mathrm{ScSR}$ is the best image super-resolution method in these 3 methods. To 
our knowledge, ScSR which is the learning based super-resolution method, will gain a better performance than image interpolation method in most cases. But it is the most timeconsuming algorithm in these super-resolution methods.

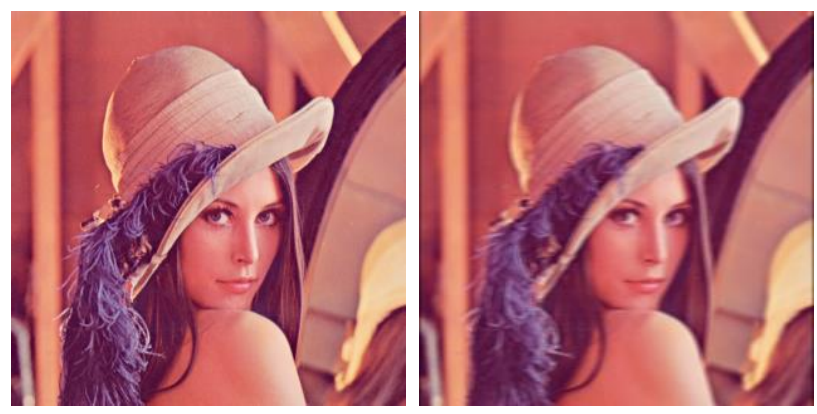

Figure 3. The Example of Original Image and LR Images

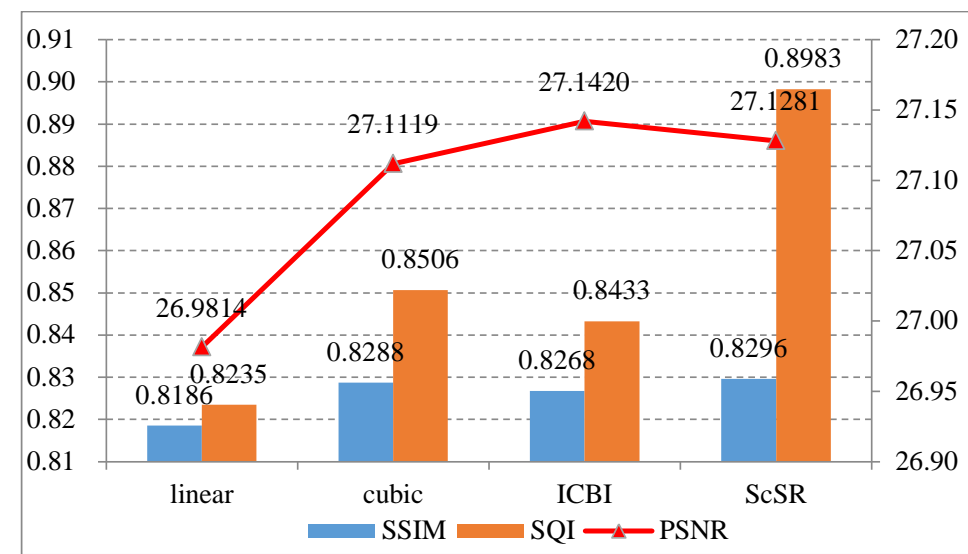

Figure 4. The Average Score of PSNR, SSIM and SQI

\subsection{Experiment II}

In this experiment, we choose 2 natural images to implement our test. As Figure.5 shows, the left image is an out-door scene named "Beach", while the right image is named "Indoor". The sizes of these images are $128^{*} 128$. We can see that Indoor is more blur than Beach.

The image super-resolution algorithms are applied to these two natural images and then we calculate SQI of each HR image, as shown in Table 2.

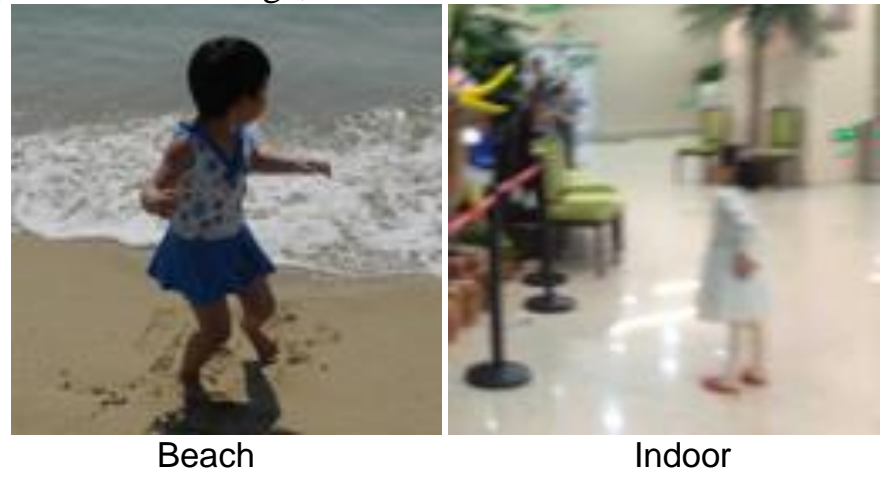

Figure 5. The Natural Images for Experiment II

In Table 2, the SQI values of Beach are higher than these of Indoor which means the SQI also can be used as the metric of super-resolution images which are reconstructed 
from different LR images. As we can find, for the image "beach", the difference between the worst and the best SQI score of image super-resolution method is 0.0581 . But for the image "indoor", the difference between the worst and the best SQI score of image superresolution method is 0.0816 which is bigger than the former. So, this result means the learning-based super-resolution method - ScSR will achieve a much better performance than the interpolation methods when the input image is a blurred one.

Table 2. The SQI of the Natural Images

\begin{tabular}{|l|l|l|l|l|}
\hline & Linear & Cubic & ICBI & ScSR \\
\hline Beach & 0.8484 & 0.8710 & 0.8684 & 0.9065 \\
\hline Indoor & 0.8160 & 0.8516 & 0.8618 & 0.8976 \\
\hline
\end{tabular}

\section{Conclusion}

In this paper, an objective image quality assessment for super-resolution images is proposed. Different from traditional image quality assessment methods, our algorithm is applicable to the situation where the sizes of the reference image and the super-resolution image are not the same. We not only consider the structural similarity between the reference image and the super-resolution image, but also image blur of the superresolution image. Experimental results demonstrate that the proposed algorithm can be used to assess the quality of super-resolution images.

\section{Acknowledgement}

This research was supported by the National Natural Science Foundation of China (No. 61461021, No. 61462031), The Key Academic Leader Plan in Jiangxi Province (No. 20133BCB22005) and the Foreign Cooperation Foundation from the science and Technology Department of Jiangxi Province (No. 20151BDH80003, 20141BDH80003), by the Natural Science Foundation of Jiangxi Province (No. 20151BAB207033), and by the Project of the Education Department of Jiangxi Province (No. KJLD14031).

\section{References}

[1] R. Y. Tsai and T. S. Huang, "Multiframe image restoration and registration," In: Huang, T.S. (ed.) Advances in Computer Vision and Image Processing, JAI Press Inc., London. (1984).

[2] N. Xiong, A.V. Vasilakos, L.T. Yang, L. Song, Y. Pan, R. Kannan and Y. Li, "Comparative analysis of quality of service and memory usage for adaptive failure detectors in healthcare systems", IEEE Journal on Selected Areas in Communications, vol.27, no.4, (2009) pp. 495-509,.

[3] N. Xiong, X. Défago, X Jia and Y. Yang, "Design and analysis of a self-tuning proportional and integral controller for active queue management routers to support TCP flows", INFOCOM, (2006).

[4] N. Xiong, Xiaohua Jia, L. T. Yang, A. V. Vasilakos, Y. Li and Y. Pan, "A distributed efficient flow control scheme for multirate multicast networks", IEEE Transactions on Parallel and Distributed Systems, vol.21, no.9, (2010) Febuary, pp. 1254-1266.

[5] Y. Zhou, Y. Zhang, H. Liu, N. Xiong and A. V. Vasilakos, "A Bare-Metal and Asymmetric Partitioning Approach to Client Virtualization", IEEE Transactions on Services Computing, vol.7, no. 1, (2014) Jan.March, pp. 40-53,

[6] M. Irani and S. Peleg, "Improving resolution by image registration," Cvgip Graphical Models \& Image Processing, vol. 53, no. 91, (1991), pp. 231-239.

[7] C. Bishop, "Bayesian image super-resolution," Advances in Neural Information Processing Systems, (2002), pp. 1303-1310

[8] J. Yang, J. Wright, T. S. Huang and Y. Ma, "Image super-resolution via sparse representation," IEEE Trans. Image Process. 19, (2010), pp. 2861-2873.

[9] Z. Wang and A. C. Bovik, "Modern Image Quality Assessment," Synthesis Lectures on Image, Video, and Multimedia Processing, vol. 2, no.1, (2006), pp. 1-156.

[10] A. M. Eskicioglu and P. S. Fisher, "Image quality measures and their performance," IEEE Trans. Commun., vol. 43, (1995) December, pp. 2959-2965. 
[11] M. P. Eckert and A. P. Bradley, "Perceptual quality metrics applied to still image compression," Signal Processing, vol. 70, (1998) November, pp. 177-200.

[12] S. Winkler, "A perceptual distortion metric for digital color video," in Proc. SPIE, vol. 3644, (1999), pp. $175-184$.

[13] Z. Wang, A. C. Bovik and L. Lu, "Why is image quality assessment so difficult," in Proc. IEEE Int. Conf. Acoustics, Speech, and Signal Processing, vol. 4, Orlando, FL, (2002) May, pp. 3313-3316.

[14] Z. Wang, A. C. Bovik and H. R. Sheikh and E. P. Simoncelli, "Image quality assessment: from error visibility to structural similarity," IEEE Trans. Image Process, vol. 13, no. 4, (2004), pp. 600-612.

[15] D. G. Lowe, "Object recognition from local scale-invariant features," in Proc. IEEE Int. Conf. Comput. Vis., vol. 2, (1999), pp. 1150-1157.

[16] Z. Wang and E. Simoncelli, "Local phase coherence and the perception of blur," in Advances in Neural Information Processing Systems, Cambridge, MA, USA: MIT Press, vol. 16, (2004) May, pp. 1-8.

[17] C. Liu, J. Yuen and A. Torralba, "SIFT flow: Dense correspondence across different scenes and its applications," IEEE Trans. Pattern Anal. Mach. Intell., vol. 33, no. 5, (2011).

[18] Y. Fang, K. Zeng, Z. Wang, W. Lin, Z. Fang and C. W. Lin, "Objective quality assessment for image retargeting based on structural similarity," Emerging \& Selected Topics in Circuits \& Systems IEEE Journal on, vol. 4, no.1, (2014), pp. 95-105.

[19] Z. Wang and A. C. Bovik, "Mean squared error: Love it or leave it? A new look at fidelity measures," IEEE Signal Process. Mag., vol. 26, no. 1, (2009) January, pp. 98-117,

[20] P. Kovesi, "Phase congruency: A low-level image invariant," Psychol. Res., vol. 64, no. 2, (2000) December, pp. 136-148.

[21] R. Hassen, Z. Wang and M. Salama, "Image Sharpness Assessment Based on Local Phase Coherence", IEEE Transactions on Image Processing, vol. 22, no. 7, (2013), pp. 2798-2810,

[22] R. Hassen, Z. Wang and M. Salama, "No-reference image sharpness assessment based on local phase coherence measurement," in Proc. IEEE Int. Conf. Acoust. Speech Signal Process, (2010) March, pp. 2434-2437.

[23] http://decsai.ugr.es/cvg/dbimagenes/

[24] A. Temizel and T. Vlachos, "Wavelet domain image resolution enhancement", IEE Proc.-Vis. Image Signal Process., vol.153, no.1, (2006), pp. 25-29,

[25] R. G. Keys, "Cubic convolution interpolation for digital image processing," IEEE Transactions on Acoustics, Speech, and Signal Processing, vol. 29, no. 6, (1981), pp. 1153-1160,

[26] A. Giachetti and N. Asuni, "Fast artifacts-free image interpolation," In: Proceedings of the British Machine Vision Conference, Leeds, UK: BMVC, (2008), pp. 123-132.

[27] http://www.ifp.illinois.edu/ jyang29./

\section{Authors}

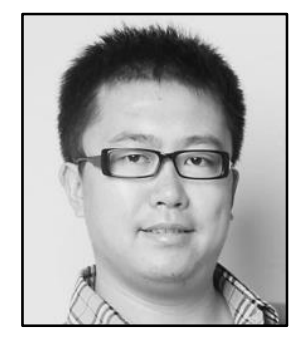

Lei Shu is a $\mathrm{PhD}$ candidate in management science and engineering, school of information technology, Jiangxi University of Finance and Economics, Nanchang, China. His research interests include image processing and machine vision.

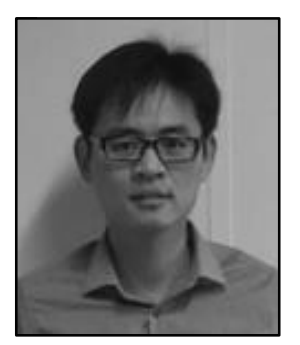

Yuming Fang received the $\mathrm{Ph} . \mathrm{D}$. degree in Computer Engineering from Nanyang Technological University, Singapore in 2013. He was a (visiting) Postdoc Research Fellow in IRCCyN lab, PolyTech' Nantes \& Univ. Nantes, Nantes, France, University of Waterloo, Waterloo, Canada and Nanyang Technological University, Singapore. His research interests include visual attention modeling, visual quality assessment, image retargeting, computer vision, 3D multimedia processing, etc. 


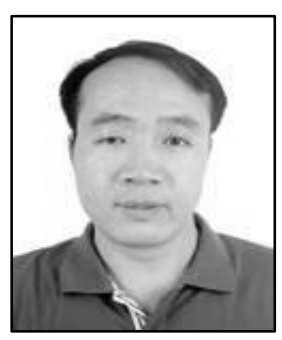

Zhijun Fang received the Ph.D. degree from Shanghai Jiaotong University, Shanghai, China. He is currently a professor in the School of Information Technology, Jiangxi University of Finance and Economics, Nanchang, China. His research interests include image processing, video coding, and pattern recognition. He was the General Chair of HHME2013 (the 9th Joint Conference on Harmonious Human Machine Environment) and the General Co-Chairs of ISITC2014 (2014 International Symposium on Information Technology Convergence).

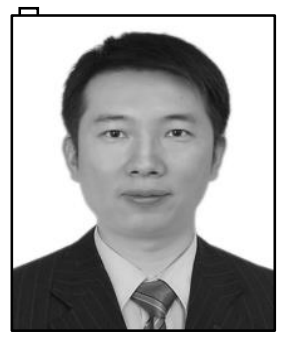

Yong Yang received the Ph.D. degree in biomedical engineering from Xi'an Jiaotong University, China, in 2005. From 2009 to 2010, he was a postdoctoral research fellow at Chonbuk National University, Republic of Korea. He is currently a Full Professor of the School of Information Technology, Jiangxi University of Finance and Economics, China. He has been awarded the title of Jiangxi Province Young Scientist since 2012. His current research interests include image processing, medical image processing and analysis, and pattern recognition.

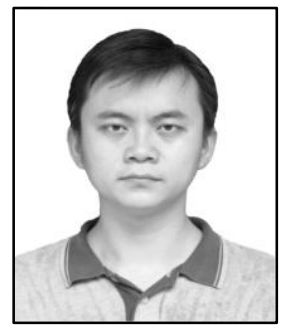

Fengchang Fei is a $\mathrm{PhD}$ candidate in management science and engineering, school of information technology, Jiangxi University of Finance and Economics, Nanchang, China. His research interests include video processing and machine vision.

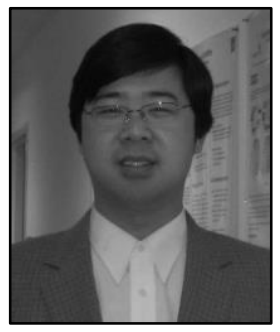

Neal N. Xiong received his both $\mathrm{PhD}$ degrees in Wuhan University (about software engineering), and Japan Advanced Institute of Science and Technology (about dependable networks), respectively. Before he attends Colorado Technical University, he worked in Wentworth Technology Institution, Georgia State University for many years. His research interests include Cloud Computing, Security and Dependability, Parallel and Distributed Computing, Networks, and Optimization Theory.

Dr./Prof. Xiong published over 100 international journal papers and over 100 international conference papers. Some of his works were published in IEEE JSAC, IEEE or ACM transactions, ACM Sigcomm workshop, IEEE INFOCOM, ICDCS, and IPDPS. He has been a General Chair, Program Chair, Publicity Chair, PC member and $\mathrm{OC}$ member of over 100 international conferences, and as a reviewer of about 100 international journals, including IEEE JSAC, IEEE SMC (Park: A/B/C), IEEE Transactions on Communications, IEEE Transactions on Mobile Computing, IEEE Trans. on Parallel and Distributed Systems. He is serving as an Editor-in-Chief, Associate editor or Editor member for over 10 international journals (including Associate Editor for IEEE Tran. on Systems, Man \& Cybernetics: Systems, Associate Editor for Information Science, Editor-in-Chief for Journal of Internet Technology (JIT), and Editor- 
in-Chief for Journal of Parallel \& Cloud Computing (PCC)), and a guest editor for over 10 international journals, including Sensor Journal, WINET and MONET. He has received the Best Paper Award in the 10th IEEE International Conference on High Performance Computing and Communications (HPCC-08) and the Best student Paper Award in the 28th North American Fuzzy Information Processing Society Annual Conference (NAFIPS2009).

Dr./Prof. Xiong is the Chair of "Trusted Cloud Computing" Task Force, IEEE Computational Intelligence Society (CIS), http://www.cs.gsu.edu/ csenxx/index-TF.html, and the Industry System Applications Technical Committee, http://ieeecis.org/technical/isatc/; He is a Senior member of IEEE Computer Society, E-mail: xiongnaixue@gmail.com 